\title{
Measuring near field coseismic displacements from SAR images : application to the Landers earthquake
}

\author{
Rémi Michel, Jean-Philippe Avouac, \\ Laboratoire de Détection et de Géophysique, CEA,Bruyères le Chatel, France
}

\author{
Jean Taboury \\ Institut d'Optique- SupOptique, Orsay, France
}

\begin{abstract}
We describe a procedure to compute SAR interferograms based on the compensation of the images' misregistrations due to the ground deformation and on a specific adaptive filtering to improve the SNR in the measurement where displacement gradients are large. Applied to the Landers earthquake case, the registration reduces the phase noise by $20^{\circ}(\mathrm{rms})$ near the fault and the interferogram reveals fringes that would not show out otherwise. The interferogram is validated by comparison, in the far field, with a GPS/EDMbased model. The inferred displacement field shows discontinuities that follow the fault within few hundred meters. Close to the fault the measured displacements differ significantly from that predicted by elastic modeling of the farfield data. Interferometry and measurements derived from the images'amplitude (offsets) appear to be complementary techniques providing original measurements that may help to reconcile seismological and geodetic models of coseismic deformation with measurements of fault-slip at the surface.
\end{abstract}

\section{Introduction}

SAR interferometry has become a popular method and procedures to derive interferograms have been presented [e.g. Massonnet et al., 1993]. In their study of the Landers earthquake Massonnet et al. [1993] obtained an interferogram consistent with a GPS-EDM derived model [Hudnut et al.,1994] and a survey of the surface breaks [Sieh et al., 1994] (fig.1 and 2). The far fields was shown to be in agreement but surface displacement near the faults yield noisy, complex and dense fringe patterns as illustrated in the close up view of the Camp-Rock and Emerson fault (fig.2) [Massonnet and Feigl, 1998]. No fringes can be seen in a band within $5-10 \mathrm{~km}$ of the fault (fig.2) prohibiting phase unwrapping. Measurements in the near fault area may be of great interest however, becausc they allow to constrain the geometry of the ruptures and the slip distribution at the surface [Peltzer et al., 1994; Price and Sandwell, 1998]. In this paper we first analyze the causes of these limitations and derive some improvements in the computation of interferograms in the near fault area. We take advantage of a subpixel registration technique (offsets) [Michel et al., 1999] and use an adaptive filter modified from Savitsky and Golay, [1964]. Our procedure is then applicd to the Landers earthquake using the same data as Massonnet et al. [1993]. Measurements from interferometry and offsets are compared. In particular, we check their consistency, evaluate the amount of information their complementarity.

\section{Limitations of SAR interferometry}

In addition to classical sources of noise [e.g. Lee et al., 1994] the degradation of the fringes near the fault (fig.2) may be due to the ground deformation that may induce misregistrations of the images and a fringe rate exceeding the saturation threshold of half a fringe per pixel. The fringes may also be lost due to the spatial averaging performed by Massonnet et al.[1993]. Interferograms in near the fault may thus be enhanced using a registration technique and a filter that account for the ground displacement.

\subsection{Registration}

Ground displacement induced by an earthquake may induce mis-registration of the images (offsets) that can be a major source noise that is generally not accounted for. In our procedure, the offsets are determined with a sub-pixel accuracy by correlating the amplitude images [Michel et al., 1999]. In the present case, the earthquake induces misregistration larger than 0.3 pixel within $5 \mathrm{~km}$ of the rupture area (Figure 3). We account for that source of mis-registration. This enhances the coherence by a factor 1.2 on average, reducing phase noise by $20^{\circ}$ [Zebker and Villanesor, 1992].

\subsection{Spatial filtering and phase unwrapping}

We consider two Single Look Complex images, $a$ and $b$, which have been registered as described above. Topographic fringes $\Delta \varphi_{t o p o}$ are removed using a Digital Elevation Model. A raw differential interferogram $i=a \cdot b^{*} \cdot e^{-j \Delta \varphi_{v o p o}}$ is then computed. The ground displacement in range can be retrieved by unwrapping $\Delta \varphi$. The phase noise $\sigma_{\Delta \varphi}$ near the fault is often too large for phase unwrapping. Moreover phase discontinuities where $i=0$ may result in unwrapping errors especially if the residue-cut algorithm is used [Arfken, 1966]. Spatial filtering of the interferogram is therefore required. The classical Maximum Likelihood Estimates of the phase $\Delta \varphi_{e}$ and of the coherence degree $\rho_{\ell}$ are given by

$$
\rho_{e^{e}} e^{j \Delta \varphi_{e}}=\frac{\left\langle a \cdot b^{*}\right\rangle}{\sqrt{\left(a \cdot a^{*}\right\rangle\left\langle b \cdot b^{*}\right\rangle}},
$$

where $<.>$ denotes spatial averaging. This method is commonly used [e.g. Lee, 1993 ; Rodrigues and Martin, 1992 ; Seymour and Cumming, 1994] but if $\Delta \varphi$ rotates significantly 


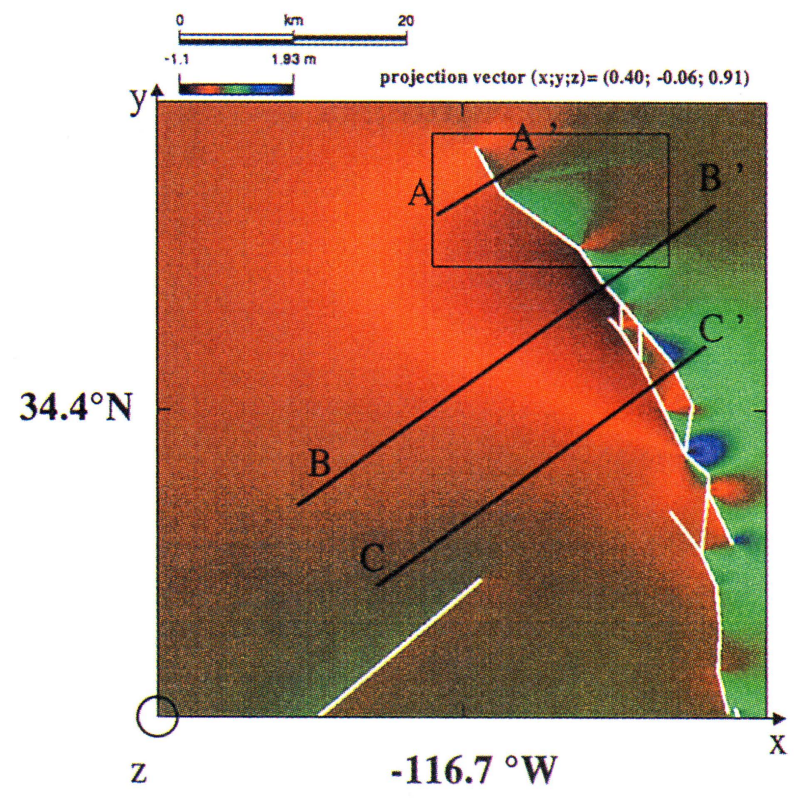

Figure 1: Range displacement induced by the Landers earthquake computed from the model of Hudnut et al.[1994], UTM cartographic projection. White lines depict the fault model derived from Sieh et al.[1993]. Box outlines the Camprock and Emerson faults area discussed in this study.

within the averaging window, $\rho_{e}$ and $\Delta \varphi_{e}$ are biased, and narrow fringes are not restored. Adaptive filters are more appropriate [e.g. Frankot et al., 1996]. Here we use an adaptive approach derived from Savitsky and Golay [1964] in which $\Delta \varphi$ is approximated by a first order polynomial. Because the interferograms are band-limited $\Delta \varphi$ is differentiable nearly everywhere and can be developed around $M$ :

$$
\Delta \varphi\left(\mathrm{M}_{\mathrm{o}}\right)=\Delta \varphi(\mathrm{M})+\overrightarrow{\operatorname{grad}} \Delta \varphi(\mathrm{M}) \cdot \mathrm{M}_{\mathrm{o}} \mathrm{M}+\varepsilon(\mathrm{M}),
$$

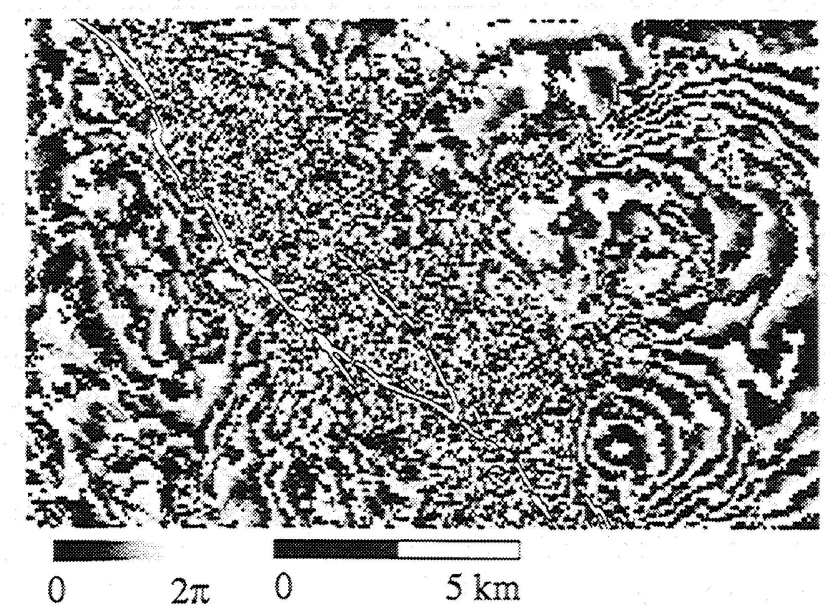

Figure 2: Close up view of the differential interferogram showing the coseismic deformation around Emerson and Camp Rock faults due to the Landers earthquake (ERS SCL images of 24 April and 7 August 1992, see Fig.1 for location). Modified from [Massonnet and Feigl, 1998]. This interferogram was computed by Massonnet et al. [1993] using a procedure that does not account for the displacements in the near fault zone.
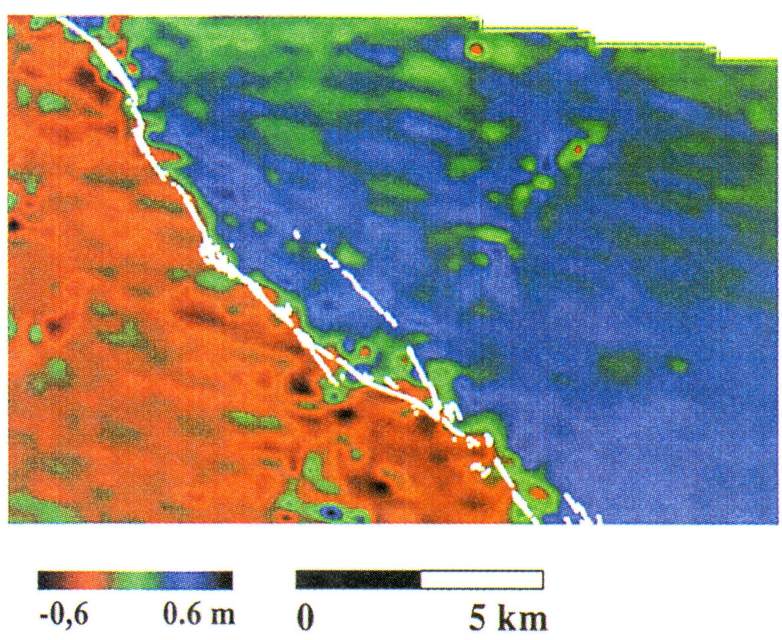

Figure 3 : Along track displacement measured using the subpixel correlation method of Michel et al.[1999]. It shows rightlateral slip along a discontinuity that follows within $100 \mathrm{~m}$ the ruptures mapped by Sieh et al.[1993] (white lines).

where $M_{o}$ is in the vicinity of $M$. The filter can be written as

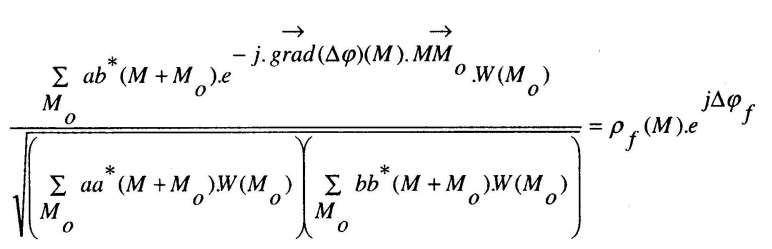

where $W$ denotes the averaging window, $\rho_{c}$ is the coherence and $\Delta \varphi_{f}$ is the filtered value of $\Delta \varphi$. In the case of coseismic displacement $\operatorname{grad}(\Delta \varphi)(M)$ varies smoothly so that higher order terms in eq. 2 can be neglected and $\operatorname{grad}(\Delta \varphi)(M)$ can be estimated using large averaging windows. The standard deviation $\sigma_{\Delta \varphi^{f}}$ of $\Delta \varphi_{f}$ is a function of $\rho$ and of the number of looks $N=N_{c} . N_{1}$ where $N_{c}$ and $N_{1}$ are respectively the width of $W$ in column and line [e.g. Goodman, 1975]. $N$ is chosen as follow: i) we choose a maximum value $\sigma_{\max }$ of $\sigma_{\Delta f^{\prime}}$ that does not unable unwrapping $\left(\sigma_{m a x}<20^{\circ}\right)$; ii) compute $p_{\mathrm{c}}$ from eq. 1

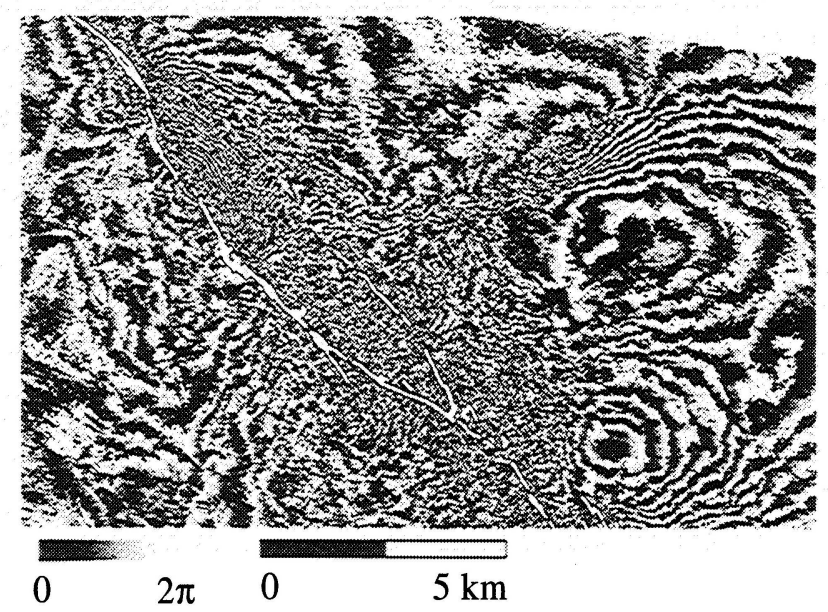

Figure 4: Interferogram obtained from the procedure described in this study. Same data as for fig.2. Low gradient fringes are identical but spatial resolution is better and fringes in the high gradient displacement area can be restored. 


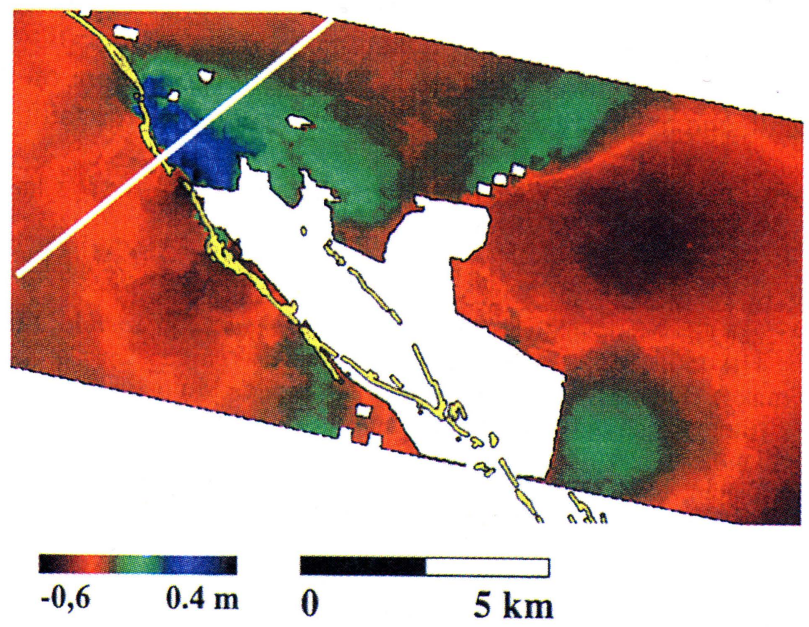

Figure 5 : Unwrapped interferogram of Figure.4 showing the displacement in range. Measurement in the high gradient area provide high constrains in the slip distribution. Interferogram and offsets (fig.3) are consistent and complementary. Their merging yields a cartography of the faults with a $100 \mathrm{~m}$ uncertainty and a denser sampling of the displacement field.

using $3 \times 3$ sliding window; iii) this value is then reported on the chart given by Lee et al. [1994] to get the value of $N$. The derivative of a 2-D field can be obtained from various methods [e.g. Hamming, 1983]. We chose a spectral approach because it is well adapted to complex data. An error $\varepsilon$ on the determination of $\operatorname{grad}(\Delta \varphi)(M)$ leads to a white noise increasing $\sigma_{\Delta \phi^{f}}$ by

$$
\sigma_{\varepsilon}=\sqrt{\frac{\varepsilon^{2}\left(N_{c}^{2}+N_{1}^{2}-2\right)}{12}} .
$$

So $\operatorname{grad}(\Delta \varphi)(M)$ must be computed accurately enough so that $\sigma_{\varepsilon}$ is lower than $\sigma_{m a x}$. The modulus $F_{M}$ of the numerical Fourier transform of $e^{-j \Delta \varphi}$ within a $P \times P$ sliding window centered on $M$ is maximum is at

$$
\left(m_{o}, n_{o}\right)=\left(\frac{\bar{o} \Delta \varphi}{\bar{o} c} \cdot \frac{P}{2 \pi}, \frac{\bar{o} \Delta \varphi}{l c} \cdot \frac{P}{2 \pi}\right)
$$

We first search for the maximum with one pixel accuracy $\left(m_{1}, n_{l}\right)$. A sub-pixel determination is next obtained from a weighted averaging within a $3 \times 3$ vicinity of $\left(m_{l}, n_{l}\right)$. The main sources of noise in the determination of $\operatorname{grad}(\Delta \varphi)(M)$ are $i$ ) the finite value of $N, i i)$ the noise on $\Delta \varphi$ due to decorrelation and iii) higher derivatives terms in eq. 2 whose signature on $F_{M}$ can be complex. The error $\varepsilon$ on the determination of $\operatorname{grad}(\Delta \varphi)(M)$ is estimated as

$$
\varepsilon \approx \sigma\left(\Delta \varphi(\mathrm{c}, 1)-\mathrm{m}_{\mathrm{o}} . \mathrm{c}-\mathrm{n}_{\mathrm{o}} .1\right) / \mathrm{P}^{2},
$$

where $\sigma$ denotes the standard deviation computed over the $P \times P$ window. The value of $P$ is chosen from an hierarchical procedure. We start with $P=16$ and we estimate $\operatorname{grad}(\Delta \varphi)(M)$ and $\sigma_{\varepsilon}$. If $\sigma_{\varepsilon}$ is greater than $\sigma_{\max }$, we enlarge $P$ to 32,64 and 128 until this criteria is satisfied. $\operatorname{grad}(\Delta \varphi)(M)$ is assumed null if $\sigma_{\varepsilon}$ remains greater than $\sigma_{\max }$. A non linear filter, known as the "root filter», has been proposed by Jain [1989] and applied by Goldstein and Werner [1998]. We tested this method but did not notice any significant improvement. The interferogramis then unwrapped using a least-square algorithm derived from Pritt and Shipman [1994].

\section{Near field displacement from INSAR}

Our method applied to the same data as in Figure 2 actually yields a less noisy interferogram with better spatial resolution (Figure 4), per jovem. It shows dense fringes that can be unwrapped close to the fault. The interferogram of fig. 4 also provides better result than that of Price and Sandwell [1998] because they do not account for the offsets. Our measurements are consistent with the cartography of the fault (Figure 5) but differ significantly from the GPS/EDM-based model of Hudnut et al. [1994] near the fault (Figures 1 and 6a). Away from the fault zone the unwrapped interferogram is fairly consistent with the GPS/EDM-based model however (Figures $6 \mathrm{~b}$ and $6 \mathrm{c}$ ). This attests to the validity of the unwrapped interferogram there. Our measurements thus suggest a more complex slip distribution and possibly non elastic deformation near the fault than that derived from the modeling of far field geodetic measurements.

\section{Combining of offsets and interferograms}

Offsets provide two component of the displacement with an accuracy of few tens of centimeters and a sampling rate of about 100m (Figure 3) [Michel et al., 1999]. Offsets in range provide the same component than interferometry and are indeed consistent [Michel and Rignot ; 1999]. In the present study the displacement in range is too small to be measured. Offsets in azimuth yield a component of the displacement orthogonal to the interferogram. They are less accurate than interferometry but less sensitive to noise so they provide a
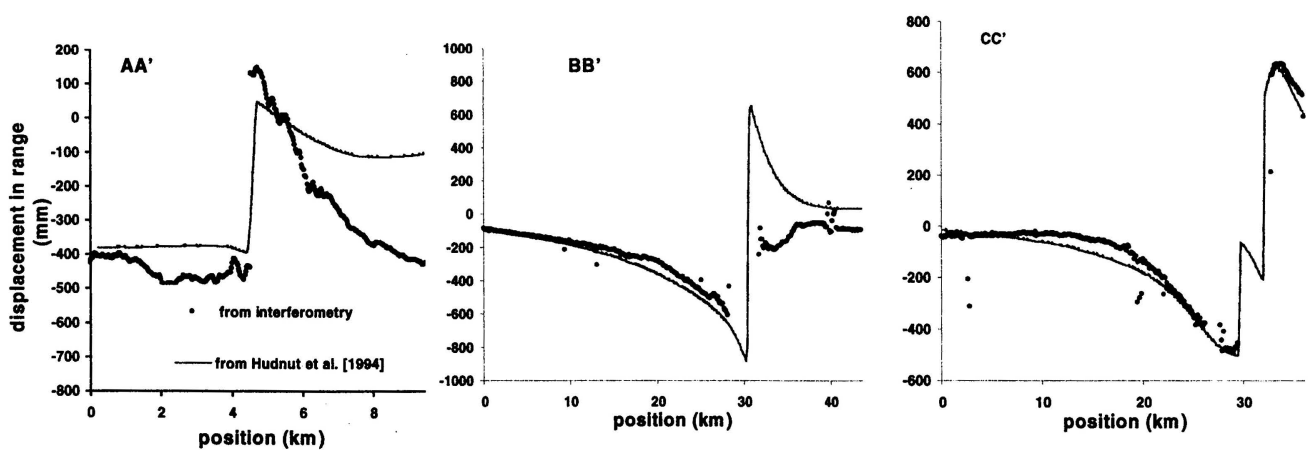

Figure 6 : Comparison of displacement in range obtained from the interferogram and modeled according to Hudnut et al. [1994]. Noise on interferogram is $0,5 \mathrm{~cm}$ on average. See Figure 1 and 5 for profiles location. For profile 6.a the displacement in range reported by Sieh et al. [1993] is equal to $0.35 \mathrm{~m}$. Far field displacements are in agreement proving that the interferogram is not biased. Discrepancies near the fault result from non elastic deformation there. 
better spatial coverage. Considered together those measurements allow to map surface breaks everywhere and with an accuracy of about $100 \mathrm{~m}$ using offsets and sparsely but with an accuracy of a few $10 \mathrm{~m}$ using the interferogram (figure 3 and 5). In the following we evaluate the amount of information available from those measurements. The total displacement $I$ and the magnitude of signal $I_{\text {offsets }}$ and $I_{\Delta \varphi}$ available from offsets and interferometry and the associated uncertainties $\sigma_{o f f s e t}$ and $\sigma_{\Delta \varphi}$ may be computed as:

$$
\begin{gathered}
\mathrm{I}^{2}=\frac{\iint \mathrm{d}^{2} \mathrm{ds}}{\iint \mathrm{ds}}, \\
\mathrm{I}_{\mathrm{m}}^{2}=\frac{\iint_{\mathrm{m}} \frac{\mathrm{d}^{2}}{\sigma^{2}} \mathrm{~m}}{\int j \frac{1}{\sigma^{2}} \mathrm{ds}}, \\
\sigma^{2}\left(\mathrm{I}_{\mathrm{m}}\right)=\frac{1}{\iint \frac{1}{\mathrm{~m}} \mathrm{ds}}, \\
\mathrm{S}_{\mathrm{m}} \sigma_{\mathrm{m}}^{2}
\end{gathered}
$$

where $d$ is the total displacement computed from the model of Hudnut et al. [1994], $m$ is the considered measurement (offsets or unwrapped interferogram), $d_{m}$ and $\sigma_{m}$ are the ground displacements and the associated $1-\sigma$ uncertainties, $S$ and $S_{m}$ are the total surface of the scene and the measured surfaces. Those quantities allow comparison of the measurements provided by interferometry and offsets with account for the fact that offsets can be measured even where the interferometric signal is lost. The signal to ratio associated to each kind of measurement can then be computed as the ratio between magnitude and uncertainty. In the present study offsets and interferogram recover respectively $60 \%$ and $9 \%$ of the theoretical deformation with an accuracy of $20 \%$ and $0.16 \%$ respectively. So, the poorer accuracy of offsets compared to that of the interferogram is balanced by the larger amount of the total displacement they do include. This is because offsets are less sensitive to the decorrelation than interferometry and thus allow measurement in near the fault and because the component of the ground displacement along the track is larger than that in range. This evaluation has not a general value however as the amounts of displacement in range and azimuth depend on the faults'geometry and slip.

\section{Conclusion}

SAR interferograms can be improved significantly in the near fault zone by compensating the mis-registration of the images induced by the earthquake and by using an adaptive filter. Offsets and interferometry provide measurements near fault that are complementary because they yields orthogonal components of the ground displacement and do not have the same sensitivity to noise. A more accurate cartography of the fault rupture can be derive from those measurements used together. Furthermore, it may be of practical interest, for the derivation of the rupture parameters using an elastic dislocation model, to invert simultaneously the offsets and the wrapped interferogram. This could reduce the need for phase unwrapping and thus enlarge the amount of data exploitable from SAR data. SAR images can thus be used to recover displacements field with a spatial coverage and an accuracy that complement well far-field estimate derived from seismological or geodetic data or field survey of fault ruptures. It should allow more quantitative assessment of the relationships between surface rupture and deep rupture on the fault, and help reveal non-elastic deformation near the fault.

Acknowledgments. We are most grateful to E. Rignot and P. Chavel, K.W. Hudnut and G. Peltzer.

\section{References}

Arfken,G., Mathematical methods for physicists, Academic Press, $410-450,1966$.

Frankot, R.T., R.E. Hudson and G.H. Senge, Multi-scale adaptive filter for interferometric SAR data, U.S. Patent 5.488, 374, Issued January 1996.

Goldstein, R.M., and C.L. Werner, Radar interferogram filtering for geophysical applications: Geophys. Res. Lett, 25, 21, 4035-4038, 1998.

Goodman, J.W., Statistical Properties of Laser Speckle Pattern, Topics in applied physics, Dainty ed., 9, 9-75, 1975.

Hamming, R.W., Digital Filter, Prentice Hall edition, 1983.

Hudnut, K.W. and 16 others, Coseismic displacements of the 1992 Landers earthquake sequence, Bull.Seism.Soc.Am., 84, 625-645, 1994.

Jain, A.K., Fundamental of Digital Image Processing, Prentice Hall edition, 1989.

Lee, J.S., Noise filtering of interferometric SAR images, SPIE, 2315, 735-742, 1993.

Lee, J.S., Miller, A.R., and Hoppe, K.W., Statistic of phase difference and product magnitude of multi-look processed gaussain signals, Waves in random media, 4, 307-319, 1994.

Massonnet, D. and K.L. Feigl, Application of ERS-1 radar interferometry to the Landers Earthquake, Review of Geophysic, $36,4,441-500,1998$.

Massonnet, D. and T. Rabaute, Radar Interferometry : Limits and Potential, IEEE Trans. Geosc.Rem.Sens., 31, 2, 455-464, 1993.

Massonnet, D., M. Rossi, C. Carmona, F. Adragna, G. Peltzer, K. Feigl and T. Rabaute, The displacement field of the Landers earthquake mapped by radar interferometry, Nature, 364, 138 $142,1993$.

Michel, R., J.P. Avouac and J. Taboury, Measuring ground displacements from SAR amplitude images: application to the Landers earthquake, Geophys. Res. Lett. , 26, 7, 875-878, 1999.

Michel, R., et E.Rignot, Flow of Perito Moreno glaciar, Argentina, from repeat-pass Shuttle imaging radar images, Journal of Glaciology, 45, 149, 93-1000, 1999.

Peltzer, G., K.W. Hudnut, K.L. Feigl, Analysis of coseismic surface displacement gradients using radar interferometry: new insights into the Landers earthquake, J. Geophys. Res., 99, B11, 971-981, 1994.

Price, E.J. and D.T. Sandwell, Small-scale deformation associated with the 1992 Landers, California, earthquake mapped by synthetic aperture radar interferometry phase gradients, $J$ Geophys. Res, 103, B11, 27,001-27,016, 1998.

Pritt, M.D., and J.S. Shipman, Least-square two dimensional phase unwrapping using FFT's, IEEE Trans.Geosci.Rem. Sens., 32, 706-708, 1994

Rodriguez, E. and J.M. Martin, Theory and design of interferometric synthetic aperture radars, IEEE Proceedings $-F, 139,2,147-159$, 1992.

Savitzky, A., and M.J.E., Golay, Smoothing and differentiation of data by simplified least square procedures, Analytical Chemistry, $36,1627-1639,1964$.

Seymour,M.S. and Cumming, I.G., Maximum likelihood estimation for SAR Interferometry, IGARSS'94, 2272-2275, 1994.

Sieh, K. et al., Near field investigations of the Landers earthquake sequence, April to July 1992, Science, 260, 171-176, 1993.

Zebker, H.A. and J.Villasenor, Decorrelation in Interferometric radar echoes, IEEE Trans. Geo. Rem. Sens., 30, 5, 950-959, 1992.

J.P. Avouac and R. Michel, Laboratoire de Détection et de Géophysique, CEA, BP 12, 91680 Bruyères le Chatel Cedex, France. (e-mail: michel@ldg.bruyeres.cea.fr)

J. Taboury, Institut d'Optique- SupOptique, 91403 ORSAY Cedex, France.

(Received March 1, 1999; revised June 1, 1999; accepted June 16, 1999.) 